\title{
EUROPEAN GROUND MOTION SERVICE (EGMS)
}

\author{
Mario Costantini, ${ }^{1}$ Federico Minati, ${ }^{1}$ Francesco Trillo, ${ }^{1}$ Alessandro Ferretti, ${ }^{2}$ \\ Fabrizio Novali, ${ }^{2}$ Emanuele.Passera, ${ }^{2}$ John Dehls, ${ }^{3}$ Yngvar Larsen, ${ }^{4}$ Petar Marinkovic, ${ }^{5}$ \\ Michael Eineder, ${ }^{6}$ Ramon Brcic, ${ }^{6}$ Robert Siegmund, ${ }^{7}$ Paul Kotzerke, ${ }^{7}$ Markus Probeck, ${ }^{7}$ \\ Ambrus Kenyeres, ${ }^{8}$ Sergio Proietti, ${ }^{1}$ Lorenzo Solari,,${ }^{9,10}$ Henrik S. Andersen ${ }^{10}$ \\ ${ }^{1}$ e-GEOS, an Italian Space Agency and Telespazio company, Rome, Italy (mario.costantini@e-geos.it) \\ ${ }^{2}$ TRE Altamira, Milan, Italy (alessandro.ferretti@tre-altamira.com) \\ ${ }^{3}$ Geological Survey of Norway, Trondheim, Norway (john.dehls@ngu.no) \\ ${ }^{4}$ NORCE, Troms $\varnothing$, Norway (ynla@ norceresearch.no) \\ ${ }^{5}$ PPO.labs, The Hague, Netherlands (petar.marinkovic@ ppolabs.com) \\ ${ }^{6}$ German Aerospace Center (DLR), Weßling, Germany (michael.eineder@dlr.de) \\ ${ }^{7}$ GAF AG, Munich, Germany (robert.siegmund@gaf.de) \\ ${ }^{8}$ Satellite Geodetic Observatory, Budapest, Hungary (ambrus.kenyeres@ sgo-penc.hu) \\ ${ }^{9}$ CTTC, Castelldefels, Spain (lorenzo.solari@ cttc.cat) \\ ${ }^{10}$ European Environment Agency, Copenhagen, Denmark (henrik.andersen@eea.europa.eu)
}

\begin{abstract}
Interferometric processing of a time series of acquisitions from synthetic aperture radar (SAR) satellites makes it possible to detect and measure ground motion phenomena, typically caused by landslides, subsidence, earthquakes or volcanic activity, with millimeter-scale precision. This enables, for example, monitoring of the stability of slopes, mining areas, buildings and infrastructures. This work presents the European Ground Motion Service (EGMS), funded by the European Commission as an essential element of the Copernicus Land Monitoring Service (CLMS). The EGMS constitutes the first application of the interferometric SAR (InSAR) technology to high-resolution monitoring of ground deformations over an entire continent, based on fullresolution processing of all Sentinel-1 (S1) satellite acquisitions over most of Europe (Copernicus Participating States). Upscaling from existing national precursor services to pan-European scale is challenging. The EGMS will employ the most advanced persistent scatterer (PS) and distributed scatterer (DS) InSAR processing techniques in combination with a high-quality Global Navigation Satellite System (GNSS) model to calibrate the ground motion products. To foster as wide usage as possible, the EGMS will also provide tools for visualization, exploration, analysis and download of the ground deformation products, as well as elements to promote best practice applications and user uptake.
\end{abstract}

Index Terms - SAR interferometry, InSAR, EGMS

\section{INTRODUCTION}

Spaceborne Interferometric synthetic aperture radar (InSAR) technology has been developed during the past three decades as a powerful instrument to measure, with millimeter-scale precision, ground motion caused by phenomena such as subsidence, landslides, tectonic effects, earthquakes or volcanic phenomena, and so to monitor, e.g., the stability of slopes, mining areas, buildings and infrastructures.

Starting with the C-band ERS mission, with the systematic acquisition of thousands of interferometric data-stacks all over the world, up to the recent X-band SAR missions COSMO-SkyMed and TerraSAR-X, with the capability of measuring surface deformations at very high spatial resolution and detail, satellite InSAR has become an indispensable technology for mapping and monitoring surface deformation phenomena over wide areas - at a fraction of the cost of conventional geodetic observations and without having to install any instruments on the ground.

The Sentinel-1 (S1) satellite mission launched in 2014, designed to systematically collect interferometric C-band SAR image stacks with global coverage at short revisit time, finally makes it possible to monitor ground deformation over entire continents with a temporal resolution of 6 days and a spatial resolution in the order of 10 meters.

The Copernicus European Ground Motion Service (EGMS) will process all S1 data over most of Europe (Copernicus Participating States) (Fig. 1) into high-resolution annually updated consistent ground motion maps and will make these publicly available. EGMS will emply persistent scatterer (PS) and distributed scatterer (DS) InSAR processing techniques [1], [2], [3], [4], [5]. Whereas low- 


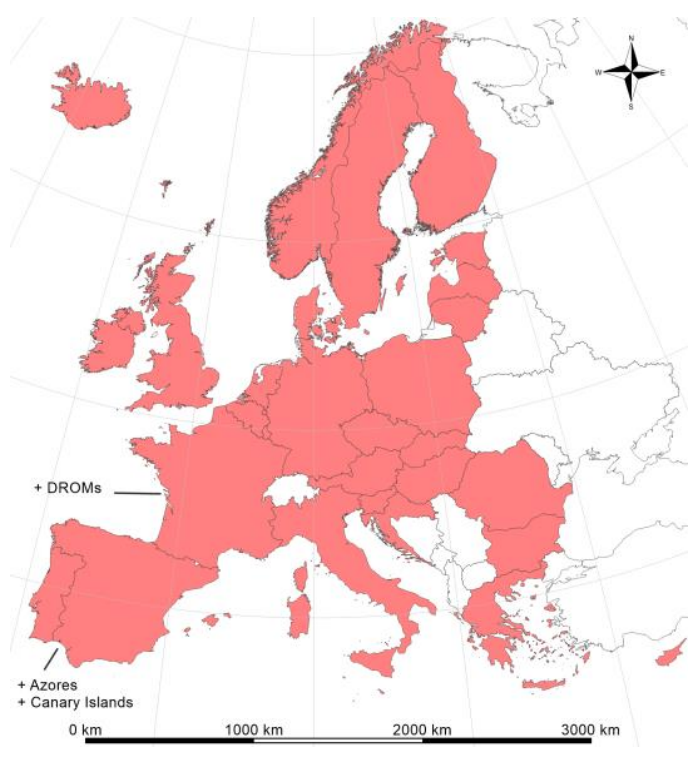

Fig. 1: EGMS initial coverage (including also French Overseas Departments and Regions).

resolution InSAR datasets over Europe have recently been proposed [6], full-resolution processing is more complex, potentially revealing errors otherwise disguised or suppressed, but in return providing the highest level of detail currently possible. The EGMS represents the first challenging application of InSAR technology to spatially and temporally high-resolution monitoring of ground deformation over an entire continent in a harmonized and continuous way.

\section{EGMS IN A NUTSHELL}

The EGMS constitutes the first application of the InSAR technology to high-resolution monitoring of ground deformations over an entire continent, based on fullresolution processing of all Sentinel-1 (S1) satellite acquisitions over most of Europe. All countries contributing to Copernicus will be covered during the first end-to-end implementation and operation of the service. This area may be extended by future realizations to include more European countries.

The EGMS will add a new and unique European-wide geospatial layer to the Copernicus Land Monitoring Service (CLMS) portfolio, dedicated to millimeter-scale measurement of ground motion caused by phenomena such as subsidence, landslides, tectonic effects, earthquakes or volcanic phenomena, with impacts on the stability of slopes, mining areas, buildings and infrastructures, and enabling further value-adding through both public and private sector downstream applications.

In order to meet the challenging project objectives, the ORIGINAL (OpeRatIonal Ground motion INsar Alliance) consortium was formed, composed of the four companies e-GEOS, TRE Altamira, NORCE and GAF, and including as subcontractors the German Aerospace Center (DLR), the

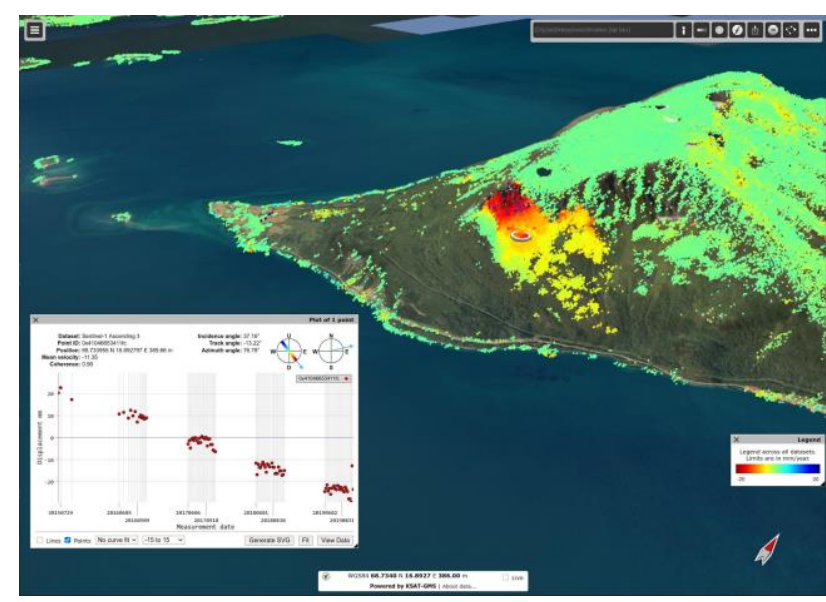

Fig. 2: Portal of the Norwegian Ground Motion Service: the EGMS portal will be built on the same technology.

Geological Survey of Norway (NGU), the Hungarian Satellite Geodetic Observatory (SGO), and the companies PPO.labs and Nhazca. By leveraging their broad experience in creating national services, the partners will build a single coherent and homogeneous service with a streamlined user experience aimed at fostering the uptake of the service and the integration of ground motion data into the operational workflows of public authorities and commercial users.

All partners will operate their own well-proven processing chains, based on advanced PS and DS InSAR processing techniques. Overlaps between adjacent processing areas will be used to check and ensure seamless harmonization between the adjacent S-1 tracks and the different processing chains.

Moreover, a high-quality Global Navigation Satellite System (GNSS) model will be used to tie the ground motion products to an established geodetic reference frame (ETRF2014). In fact, the consortium can count on leading GNSS experience. This will also provide a unique link between the EGMS and the GNSS community.

Furthermore, having the future EGMS provided by the same organizations currently providing national ground motion products will increase the level of trust from endusers and ensure consistency between the EGMS and national/regional services.

The first baseline product, using S1 data from 2015-2020, will be delivered at the beginning of 2022 and will be followed by annual updates. The products will be consistent, standardized, interoperable, and harmonized across national borders to provide one homogeneous European dataset. In order to foster as wide a usage as possible by the growing Copernicus user community and the public at large, the EGMS will additionally provide tools for visualization, exploration, analysis and download as well as elements to promote best practice applications and user uptake. 


\section{PRODUCTS}

Different types of products will be realized and provided by the EGMS:

- Level 2a (L2a): InSAR displacement data in ascending and descending geometry, which are precise but considered low-level since projected in the satellite lineof-sight. Location data (coordinates) and quality measures are provided per measurement point (MP). Relative products are generated by independent processing of single SAR stacks with a local reference point. These relative products are suited for the measurement and interpretation of predominantly local movements.

- Level 2b (L2b): Like L2a, but calibrated with a GNSS reference network to provide products in a common reference frame, so that measurements are no longer relative to a reference MP. L2b products are more intuitively interpretable and more compatible with other geophysical data and market sectors, plus better capturing large-scale phenomena.

- Level 3 (L3): Comprises horizontal and vertical ground deformation, derived from multiple L2b products from complementary geometries. It will be provided in two geospatial layers: Vertical and East-West components of displacement, both anchored to the GNSS reference network in a common reference frame.

- A-EPND (Augmented EPND) and large scale GNSS deformation model: Integration of maintained long-term GNSS networks such as EPND, NGL into a model of large-scale deformation in the East, North and vertical directions, used to anchor the L2b and L3 measurements to a common reference frame.

At the beginning of 2022, the above products, generated using all S1 Interferometric Wide Swath (IWS) acquisitions from Feb. 2015 till Dec. 2020, will be made available via a dedicated EGMS portal. The products will be updated annually.

\section{METHODOLOGY}

The whole set of S1 acquisitions over the selected European countries (Fig. 1) will be be used to realize the EGMS products described in the previous section. The expected total amount of uncompressed S1 data is about 1.5 PB for the baseline analysis. Each annual update requires about 290 TB of additional input data.

In order to maximize the information content by obtaining measurements in both urban and rural areas, the identification of measurement points (MPs) and the reconstruction of the associated displacement history will rely on a hybrid PS/DS analysis [1], [2], [3], [4], [5]. Depending on the density of PS and their average phase coherence, DS will be identified and used as MP when useful to increase the spatial coverage of the data in non-urban areas.

Although the original PS technique [1] was based on a linear model for describing the motion of the radar targets, advanced InSAR techniques will be applied [2], [5], allowing users to highlight MP exhibiting more complex displacement time series (e.g. seasonal components, acceleration, etc.). Of course, the massive data processing associated with EGMS will call for a number of trade-offs between accuracy and computational load, which is inherent in every large-scale application of remote sensing data. In general, we expect the number and the quality of MP to increase with time, as algorithms will be further improved, and computational resources will become more and more affordable.

Since InSAR is a technique providing relative measurements (i.e. it measures displacements between points belonging to the same satellite swath), external information is needed to reliably reference L2a data to an "absolute" geodetic reference frame. Considering the continental extent of the EGMS service, the only available external information source of sufficiently high quality and consistency is from systematically maintained long-term GNSS networks. For the purposes of the EGMS, a low-resolution gridded 3D velocity model, based on integrating reliable GNSS stations from EPND, NGL, etc., will be developed on a 50-km grid.

For producing calibrated L2b products, the reference velocity model obtained from GNSS data is transformed to the reference frame of the SAR (ETRF2014), projected to the radar line-of-sight, interpolated at each MP, and used to provide the a priori information needed to harmonize different reference points, effectively calibrating the L2a products. Low-frequency GNSS data will be useful also to compensate for possible atmospheric leakage impacting on the average displacement rates. Therefore, large-scale, GNSS-compatible, displacement data are generated using a standard approach, valid and applicable to all interferometric techniques used for producing the EGMS service.

Special attention will be paid for the calibration of the area where: (a) the density of GNSS stations is low and the $a$ posteriori covariance of GNSS data will not allow a precise calibration; (b) the area is affected by strong non-linear deformation phenomena (possibly induced by seismic events) where low-frequency, large scale, velocity models can merely provide a first-order correction. In both cases, the consortium will rely on SAR data acquired over the same area from different acquisition geometries (overlapping satellite tracks, as well as ascending and descending passes) to come up with the best estimation of the local deformation field.

The combination of different observation geometries to retrieve both vertical and horizontal components of the displacement vector is a well-proven technique [7] and L3 EGMS products are expected to provide an easy-to-use, GNSS compatible, dataset, providing users with true vertical and east-west displacement data. Of course, the availability of L3 data will depend on the availability of MP in L2b ascending and descending datasets. In fact, in mountainous 
areas or, in general, in regions characterized by rough topography, shadowing, foreshortening and layover effects can prevent the estimation of $2 \mathrm{~d}$ displacement measurements. However, L3 data, wherever available, are expected to provide a "GPS-like" information layer of immediate use, also for non-expert users.

Quality control procedures will be based on a set of check lists to be followed by all InSAR providers. Apart from standard QC based on phase coherence and the amplitude stability index, ad hoc procedures will be adopted to take advantage of overlapping tracks, which will allow production engineers to run internal cross-check procedures. Moreover, a statistical analysis of MP density over different classes of terrain (based on Copernicus CORINE land use data), will allow operators to spot anomalies and adopt any relevant countermeasures.

\section{PRODUCT ARCHIVE AND DISSEMINATION}

The EGMS Dissemination System is the main tool enabling the use of EGMS data by users. Based upon the same technology currently in use in the Norwegian Ground Motion Service (Fig. 2), the system will provide InSAR product visualisation, simple data interrogation and manipulation, user-determined download and basic webGIS functionality. Search and retrieve functionality will be provided for users who need to work with data products directly in their own GIS systems or other data analysis software. Data download will be available to registered users through an interactive web interface, and an API following one or more open standards, e.g. OpenSearch and oData.

\section{USER UPTAKE AND AWARENESS RAISING}

EGMS is a new and innovative Copernicus service, and it is of utmost importance to raise awareness about the InSAR products and their potential use in downstream user's applications. Therefore, the project's specific goal is to address the main target user communities and stimulate and support users in the use and integration of the EGMS products into their application/production workflows. To this aim, information and user engagement activities are planned already in 2021 with demonstrational products. Then, starting from 2022, when the validated baseline products will be available, targeted workshops, webinars and showcases will be organized at major Copernicus events and at National User Forums.

\section{CONCLUSIONS}

This work presents the European Ground Motion Service (EGMS), funded by the European Commission as an essential element of the Copernicus Land Monitoring Service (CLMS).

The EGMS constitutes the first application of the InSAR technology to high-resolution monitoring of ground deformations over an entire continent, based on full- resolution processing of all Sentinel-1 (S1) satellite acquisitions over most of Europe (Copernicus Participating States). EGMS will monitor, with millimeter-scale precision, ground motion caused by phenomena such as subsidence, landslides, tectonic effects, earthquakes or volcanic phenomena, with impacts on the stability of slopes, mining areas, buildings and infrastructures.

The final version of the paper, and the presentation, the first results of the EGMS.

\section{ACKNOWLEDGEMENTS}

The EGMS project receives funding from the European Commission's Copernicus programme, under the European Environment Agency's framework service contract EEA/DIS/R0/20/011. The opinions expressed herein are only those of the authors and do not represent the official positions of the European Environment Agency.

\section{REFERENCES}

[1] Ferretti, A., Prati, C. and Rocca, F., Permanent scatterers in SAR interferometry. IEEE TGARS, 2001, 39(1), pp.8-20.

[2] A. Ferretti, C. Prati, and F. Rocca, "Non-linear subsidence rate estimation using permanent scatterers in differential SAR interferometry," IEEE TGARS, vol. 38, no. 5, pp. 2202-2212, 2000.

[3] A. Ferretti, A. Fumagalli, F. Novali, C. Prati, F. Rocca, and A. Rucci, A new algorithm for processing interferometric data-stacks: SqueeSAR. IEEE TGARS, 2011, 49(9), pp.3460-3470.

[4] M. Costantini, S. Falco, F. Malvarosa, F. Minati, F. Trillo, F. Vecchioli, "Persistent scatterer pair interferometry: approach and application to COSMO-SkyMed SAR data," IEEE JSTARS, vol. 7, no. 7, pp. 2869-2879, 2014.

[5] M. Costantini, F. Malvarosa, F, Minati, "A General Formulation for Redundant Integration of Finite Differences and Phase Unwrapping on a Sparse Multidimensional Domain," IEEE TGARS, vol. 50, no. 3, pp. 758-768, 2012.

[6] Lanari, R., Bonano, M., Casu, F., De Luca, C., Manunta, M., Manzo, M., Onorato, G., Zinno, I., "Automatic Generation of Sentinel-1 Continental Scale DInSAR Deformation Time Series through an Extended P-SBAS Processing Pipeline in a Cloud Computing Environment," Remote Sens. 2020, 12(18), 2961.

[7] Bürgmann, R., Hilley, G., Ferretti, A. and Novali, F., 2006. Resolving vertical tectonics in the San Francisco Bay Area from permanent scatterer InSAR and GPS analysis. Geology, 34(3), pp.221-224. 